\title{
High efficiency computation of the variances of structural evolutionary random responses
}

\author{
J.H. Lin ${ }^{\mathrm{a}, *}$, W.X. Zhong ${ }^{\mathrm{a}}$, W.S. Zhang ${ }^{\mathrm{b}}$ and \\ D.K. Sun ${ }^{\mathrm{b}}$

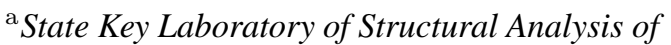 \\ Industrial Equipment, Dalian University of \\ Technology, Dalian 116023, China \\ ${ }^{\mathrm{b}}$ Dept of Civil and Structural Engineering, Hong \\ Kong Polytechnic University, Kowloon, Hung Hom, \\ Hong Kong
}

Received 23 April 1999

Revised 14 April 2000

For structures subjected to stationary or evolutionary white/ colored random noise, their various response variances satisfy algebraic or differential Lyapunov equations. The solution of these Lyapunov equations used to be very difficult. A precise integration method is proposed in the present paper, which solves such Lyapunov equations accurately and very efficiently.

\section{Introduction}

Many engineering structures, e.g. bridges, tallbuildings, TV towers, offshore platforms, are very sensitive to the ambient random loads such as windgusts, earthquakes, irregular waves, etc. The analysis methodology for these problems have received much attention, and many contributions have been published (e.g. $[1,2,8,9,14])$. For most problems, the variance analysis is of major interest. However, if the structures are subjected to evolutionary random excitations, such analysis is quite difficult. Recently, Lin et al. [3-7] developed the pseudo excitation method (PEM) which has enabled the computation of such problems rather conveniently. Unfortunately, for structures subjected to wide band excitations, a great number of frequency

${ }^{*}$ Corresponding author: Prof. Lin Jiahao, Research Institute of Engineering Mechanics, Dalian University of Technology, Dalian 116023, P. R. China. Fax: +86 411 4709295; E-mail: jhlin@ dlut. edu.cn. points still have to be taken in order to obtain accurate variances, and the computation would still be rather time-consuming. Further improvement is still required.

In past few years, Zhong, X.N. [10] derived the closed solutions of the algebraic and differential Lyapunov equations based on the precise numerical computation of the exponential matrix $\exp ([\mathrm{A}] \mathrm{t})$ proposed by Zhong, W.X. [11-13]. In the present paper, these methods have been combined and extended to the solution of the above mentioned variances. Two examples are given which show that the proposed method is not only accurate, but also extremely efficient.

\section{Differential Lyapunov equation}

The equations of motion of an $\mathrm{n}$ DOF (degrees of freedom) structure subjected to an evolutionary random excitation $\{f(t)\}$ can be expressed as

$$
\begin{aligned}
{[M]\{\ddot{y}\}+[C]\{\dot{y}\}+[K]\{y\} } & =\{f(t)\} \\
& =g(t)\{x(t)\}
\end{aligned}
$$

Eq. (1) can be expressed as

$$
\begin{aligned}
\left\{\begin{array}{l}
\dot{y} \\
\ddot{y}
\end{array}\right\}= & {\left[\begin{array}{cc}
0 & I \\
-M^{-1} K & -M^{-1} C
\end{array}\right]\left\{\begin{array}{l}
y \\
\dot{y}
\end{array}\right\} } \\
& +\left[\begin{array}{c}
0 \\
g(t) I
\end{array}\right]\{x(t)\}
\end{aligned}
$$

or

$$
\{\dot{z}\}=[A]\{z\}+[G]\{x\}
$$

where $[M],[C]$ and $[K]$ are the mass, damping and stiffness matrices of the structure, $\{y\},\{\dot{y}\}$ and $\{\ddot{y}\}$ are its displacement, velocity and acceleration vecters, $\{x(t)\}$ is a stationary Gausian white noise random process vector; $g(t)$ is a slowly varying modulation function, and $[I]$ is a unit matrix of order $\mathrm{n}$.

If the RHS (right hand side) of Eq. (1) is set to zero, the term $M[G]\{\chi\}$ in Eq. (3) will vanish, and so its solution would be $[3,4]$

$$
\left[\Phi\left(t, t_{0}\right)\right]=\left[\Phi\left(t-t_{0}\right)\right]=\exp \left([A]\left(t-t_{0}\right)\right)
$$


If the RHS of Eq. (1) is not zero, the solution of Eq. (3) would be

$$
\begin{aligned}
\{z(t)\}= & {\left[\Phi\left(t, t_{0}\right)\right]\left\{z_{0}\right\} } \\
& +\int_{t_{0}}^{t}[\Phi(t, s)]\{f(s)\} d s
\end{aligned}
$$

in which $\left\{z_{0}\right\}$ is the values of $\{z(t)\}$ at the initial time $t_{0}$. Usually, the initial conditions can be regarded as uncorrelated with the excitations, so that Eq. (5) leads to the covariance matrix

$$
\begin{aligned}
{\left[R_{z z}\left(t_{1}, t_{2}\right)\right]=} & E\left\lfloor\left\{z\left(t_{1}\right)\right\}\left\{z\left(t_{2}\right)\right\}^{T}\right\rfloor \\
= & {\left[\Phi\left(t_{1}, t_{0}\right)\right]\left[R_{z z}\left(t_{0}, t_{0}\right)\right]\left[\Phi\left(t_{2}, t_{0}\right)\right]^{T} } \\
& +\int_{t_{0}}^{t_{1}} d s_{1} \int_{t_{0}}^{t_{2}}\left[\Phi\left(t_{1}, s_{1}\right)\right]\left[G\left(s_{1}\right)\right] \\
& E\left[\left\{x\left(s_{1}\right)\right\}\left\{x\left(s_{2}\right)\right\}^{T}\right] \cdot\left[G\left(s_{2}\right)\right]^{T} \\
& {\left[\Phi\left(t_{2}, s_{2}\right)\right]^{T} d s_{2} }
\end{aligned}
$$

For the stationary white noise process $\{x(t)\}$ of which the intensity function is $[D]$, its auto-correlation function is

$$
\begin{aligned}
{\left[R_{x x}\left(s_{1}, s_{2}\right]\right.} & =E\left[\left\{x\left(s_{1}\right)\right\}\left\{x\left(s_{2}\right)\right\}^{T}\right] \\
& =[D] \delta\left(s_{2}-s_{1}\right)
\end{aligned}
$$

That makes Eq. (6), when $t_{1}=t_{2}=t$, become

$$
\begin{aligned}
E\left[\{z(t)\}\{z(t)\}^{T}\right]= & {\left[\Phi\left(t, t_{0}\right)\right]\left[R_{z z}\left(t_{0}, t_{0}\right)\right] } \\
& {\left[\Phi\left(t, t_{0}\right)\right]^{T}+\int_{0}^{t}[\Phi(t, s)] } \\
& {[G(s)][D][G(s)]^{T} } \\
& {[\Phi(t, s)]^{T} d s }
\end{aligned}
$$

Denote $[P]=E\left[\{z(t)\}\{z(t)\}^{T}\right]$, its first order derivative about time $t$ is

$$
\begin{aligned}
{[\dot{P}]=} & E\left[\{\dot{z}(t)\}\{z(t)\}^{T}\right]+E\left[\{z(t)\}\{\dot{z}(t)\}^{T}\right] \\
= & {\left[\dot{\Phi}\left(t, t_{0}\right)\right]\left[R_{z z}\left(t_{0}, t_{0}\right)\right]\left[\Phi\left(t, t_{0}\right)\right]^{T} } \\
& +\left[\Phi\left(t, t_{0}\right)\right]\left[R_{z z}\left(t_{0}, t_{0}\right)\right]\left[\dot{\Phi}\left(t, t_{0}\right)\right]^{T} \\
& +\int_{t_{0}}^{t}[\dot{\Phi}(t, s)][G(s)][D][G(s)]^{T}[\Phi(t, s)]^{T} d s \\
& +\int_{t_{0}}^{t}[\Phi(t, s)][G(s)][D][G(s)]^{T}[\dot{\Phi}(t, s)]^{T} d s \\
& +[\Phi(t, t)][G(t)][D][G(t)]^{T}[\Phi(t, t)]^{T}
\end{aligned}
$$

As the principal solution of $\Phi$ satisfies

$$
\left[\dot{\Phi}\left(t, t_{0}\right)\right]=[A(t)]\left[\Phi\left(t, t_{0}\right)\right], \quad[\Phi(t, t)]=[I]
$$

That leads to the differential Lyapunov equation

$$
[\dot{P}]=[A][P]^{T}+[P][A]^{T}+[G(t)][D][G(t)]^{T}
$$

\section{Precise integration for Lyapunov equation}

Consider the homogeneous differential Lyapunov equations

$$
[\dot{P}]=[A][P]^{T}+[P][A]^{T}
$$

The values $\left[P_{0}\right]$ at initial time $t_{0}$ are known. It is readily verified that its solution is

$$
\left[P_{h}(t)\right]=[\Phi(t)]\left[P_{0}\right][\Phi(t)]^{T}
$$

Next, let

$$
[Q(s)]=[G(s)][D][G(s)]^{T}
$$

and

$$
\left[P_{s}(t)\right]=\int_{t_{0}}^{t}[\Phi(t-s)][Q(s)][\Phi(t-s)]^{T} d s
$$

Because

$$
\begin{aligned}
\frac{d}{d t}\left[P_{s}(t)\right]= & {[\Phi(0)][Q(t)][\Phi(0)]^{T} } \\
& +\int_{t_{0}}^{t}[\dot{\Phi}(t-s)][Q(s)][\Phi(t-s)]^{T} d s \\
& +\int_{t_{0}}^{t}[\Phi(t-s)][Q(s)][\dot{\Phi}(t-s)]^{T} d s \\
= & {[Q(t)]+[A]\left[P_{s}(t)\right]+\left[P_{s}(t)\right][A]^{T} }
\end{aligned}
$$

It shows that $\left[P_{s}(t)\right]$ is the particular solutions of the non-homogeneous equations (11). Therefore, the solutions of Eq. (11) with non-zero initial values should be

$$
\begin{aligned}
{[P(t)]=} & {\left[P_{h}(t)\right]+\left[P_{s}(t)\right]=[\Phi(t)]\left[P_{0}\right][\Phi(t)]^{T} } \\
& +\int_{t_{0}}^{t}[\Phi(t-s)][Q(s)][\Phi(t-s)]^{T} d s
\end{aligned}
$$

In the following, only the case with $\left[P_{0}\right]=[0]$ (when $\left.t_{0}=0\right)$ is considered. The non-zero initial-value solution can be separately solved and then be linearly added to the zero-initial-value solution in order to produce the complete solution. Obviously, at time $t$,

$$
[P(t)]=\int_{t_{0}}^{t}[\Phi(t-s)][Q(s)][\Phi(t-s)]^{T} d s
$$

Since

$$
\left[\Phi\left(t_{1}+t_{2}\right)\right]=\left[\Phi\left(t_{1}\right)\right]\left[\Phi\left(t_{2}\right)\right]
$$

therefore at time $t+\tau$ 


$$
\begin{aligned}
{[P(t+\tau)]=} & \int_{0}^{t}[\Phi(t+\tau-s)][Q(s)] \\
& {[\Phi(t+\tau-s)]^{T} d s } \\
& +\int_{t_{0}}^{t+\tau}[\Phi(t+\tau-s)][Q(s)] \\
& {[\Phi(t+\tau-s)]^{T} d s } \\
= & {[\Phi(\tau)][P(t)][\Phi(\tau)]^{T} } \\
& +\int_{0}^{\tau}[\Phi(\tau-s)][Q(t+s)] \\
& {[\Phi(\tau-s)]^{T} d s }
\end{aligned}
$$

Similarly, the double-step solution (i.e. that at time $2 t$ ) can be obtained, being

$$
\begin{aligned}
{[P(2 t)]=} & {[\Phi(t)][P(t)][\Phi(t)]^{T}+\int_{0}^{t}[\Phi(t-s)] } \\
& {[Q(t+s)][\Phi(t-s)]^{T} d s }
\end{aligned}
$$

The formulas for some simple modulation functions are given in the following. It is always assumed that when $t<0, g(t)=0$. The response variances are going to be computed at time $\tau, 2 \tau, 3 \tau, \ldots$.

\subsection{For suddenly applied white noise}

Let $g(t)=1.0$ (when $t \geqslant 0$, the RHS of Eq. (1) with this modulation function corresponds to a suddenly applied white noise. Clearly, $[G(t)]$ and $[Q(t)]$ are both constants. Denote $\left[Q(t)=\left[Q_{0}\right]\right.$, and so the solution of Eq. (18) at time $t_{k}=k \tau$ would be

$$
\begin{aligned}
{\left[P^{(0)}\left(t_{k}\right)\right]=} & \int_{0}^{t_{k}}\left[\Phi\left(t_{k}-s\right)\right]\left[Q_{0}\right] \\
& {\left[\Phi\left(t_{k}-s\right)\right]^{T} d s }
\end{aligned}
$$

So that at time $t_{k+1}=k \tau+\tau$

$$
\begin{aligned}
{\left[P^{(0)}\left(t_{K+1}\right)\right]=} & \int_{0}^{t_{k}+\tau}\left[\Phi\left(t_{k}+\tau-s\right)\right]\left[Q_{0}\right] \\
& {\left[\Phi\left(t_{k}+\tau-s\right)\right]^{T} d s } \\
= & \int_{0}^{t_{k}}\left[\Phi\left(t_{k}+\tau-s\right)\right]\left[Q_{0}\right] \\
& {\left[\Phi\left(t_{k}+\tau-s\right)\right]^{T} d s } \\
& +\int_{t_{k}}^{t_{k}+\tau}\left[\Phi\left(t_{k}+\tau-s\right)\right]\left[Q_{0}\right] \\
& {\left[\Phi\left(t_{k}+\tau-s\right)\right]^{T} d s }
\end{aligned}
$$

$$
\begin{aligned}
= & {[\Phi(\tau)] \int_{0}^{t}\left[\Phi\left(t_{k}-s\right)\right]\left[Q_{0}\right] \quad(23) } \\
& {\left[\Phi\left(t_{k}-s\right)\right]^{T} d s[\Phi(\tau)]^{T} } \\
& +\int_{0}^{\tau}[\Phi(\tau-s)][\Phi(\tau-s)]\left[Q_{0}\right] \\
& {[\Phi(\tau-s)]^{T} d s } \\
= & {[\Phi(\tau)]\left[P^{(0)}\left(t_{k}\right)\right][\Phi(\tau)]^{T} } \\
& {\left[P^{(0)}(\tau)\right] }
\end{aligned}
$$

Similarly, at time $2 t_{k}$, the double step solution is

$$
\begin{aligned}
{\left[P^{(0)}\left(2 t_{k}\right)\right]=} & {\left[\Phi\left(t_{k}\right)\right]\left[P^{(0)}\left(t_{k}\right)\right]\left[\Phi\left(t_{k}\right)\right]^{T} } \\
& +\left[P^{(0)}\left(t_{k}\right)\right]
\end{aligned}
$$

\subsection{For time dependent modulation function}

Provided $g(t)=\sqrt{t}($ when $t \geqslant 0)$, then $[Q(t)]=$ $\left[Q_{0}\right] t$. At time $t_{k}$, the variance matrix is [9]

$$
\begin{aligned}
{\left[P^{(1)}\left(t_{k}\right)\right]=} & \int_{0}^{t_{k}}\left[\Phi\left(t_{k}-s\right)\right]\left[Q_{0}\right] s \\
& {\left[\Phi\left(t_{k}-s\right)\right]^{T} d s }
\end{aligned}
$$

This matrix has the form at time $t_{k}+\tau$

$$
\begin{aligned}
{\left[P^{(1)}\left(t_{k+1}\right)\right]=} & \int_{0}^{t_{k}+\tau}\left[\Phi\left(t_{k}-s\right)\right]\left[Q_{0}\right] s \\
& {\left[\Phi\left(t_{k}-s\right)\right]^{T} d s } \\
= & {[\Phi(\tau)]\left[P^{(1)}\left(t_{k}\right)\right][\Phi(\tau)]^{T} } \\
& +\int_{0}^{\tau}[\Phi(\tau-s)]\left[Q_{0}\right] \\
& \left(t_{k}+s\right)[\Phi(\tau-s)]^{T} d s \\
= & {[\Phi(\tau)]\left[P^{(1)}\left(t_{k}\right)\right][\Phi(\tau)]^{T} } \\
& +t_{k}\left[P^{(0)}(\tau)\right]+\left[P^{(1)}(\tau)\right]
\end{aligned}
$$

and the corresponding double-step form would be

$$
\begin{aligned}
{\left[P^{(1)}\left(2 t_{k}\right)\right]=} & {\left[\Phi\left(t_{k}\right)\right]\left[P^{(1)}\left(t_{k}\right)\right]\left[\Phi\left(t_{k}\right)\right]^{T} } \\
& +t_{k}\left[P^{(0)}\left(t_{k}\right)\right]+\left[P^{(1)}\left(t_{k}\right)\right]
\end{aligned}
$$

The integration formats for other modulation functions $g(t)$, so long as they are not too complicated, can be similarly derived. Note that the above formulas are all exact. The key problem is how to ensure high precision on computers. 


\section{Precise integration of Lyapunov equation and its implementation on computers}

In order to achieve precise integration on computers, the step size $\tau$ should be divided into $2^{N}$ equal intervals, $N=20$ is usually adequate [10]. Take the values of $[\Phi(\eta)]$ and $[P(\eta)]$ at time $\eta=\tau / s^{N}$ as the initial values for the step-by-step integration. Because $\eta$ is extremely small, therefore taking only the first 3 or 4 terms in the related Taylor's expansions would achieve extremely high precision. This is the essence of the precise integration approach. The first step in implementation is to execute the double step formulas for $N$ times starting from the initial time $t=0$, to produce their values at time $\tau$. Secondly, the precise integration is executed with the same step size $\tau$ to generate the response variances at time $2 \tau, 3 \tau, \ldots$ The implementation process for $g(t)=\sqrt{t}$ is given here in detail. For other modulation functions, the processes are quite similar, and will be referred to the users.

1. Divide $\tau$ into $2^{N}$ equal parts, let $\eta=\tau / 2^{N}$, $t t=\eta$.

2. Use Taylor's expansion to compute

$$
\begin{aligned}
& {[\Phi(\eta)] \approx\left[I_{n}\right]+\left[T_{a}\right], } \\
& {\left[T_{a}\right]=[A] \eta+}([A] \eta)^{2}\left[\left[I_{n}\right]+([A] \eta) / 3\right. \\
&\left.+([A] \eta)^{2} / 12\right] / 2 \\
& {\left[P^{(0)}(\eta)\right] \approx } {\left[Q_{0}\right] \eta+\left([A]\left[Q_{0}\right]+\left[Q_{0}\right][A]^{T}\right) } \\
& \eta^{2} / 2+\left([A]^{2}\left[Q_{0}\right]+2[A]\left[Q_{0}\right][A]^{T}\right. \\
&\left.+\left[Q_{0}\right][A]^{2 T}\right) \eta^{3} / 6+\left([A]^{3}\left[Q_{0}\right](29)\right. \\
&+3[A]^{2}\left[Q_{0}\right][A]^{T}+3[A]\left[Q_{0}\right][A]^{2 T} \\
&\left.+\left[Q_{0}\right][A]^{3 T}\right) \cdot \eta^{4} / 24 \\
& {\left[P^{(1)}(\eta)\right]=} {\left[Q_{0}\right] \eta^{2} / 2+\left([A]\left[Q_{0}\right]+\left[Q_{0}\right][A]^{T}\right) } \\
& \eta^{3} / 6+\left([A]^{2}\left[Q_{0}\right]+2[A]\left[Q_{0}\right] \quad(30)\right. \\
& {\left.[A]^{T}+\left[Q_{0}\right][A]^{2 T}\right) \eta^{4} / 24 }
\end{aligned}
$$

With $N=20$, the errors due to neglecting the higher order terms in the Taylor's series are all of the order of $O\left(\eta^{5}\right)=O\left(\tau^{5} / 10^{30}\right)$, which is within the doubleprecision of available computers.

3. Repeat the following process for $N$ times, to work out the variance matrix $[P]$ at time $\tau$ :

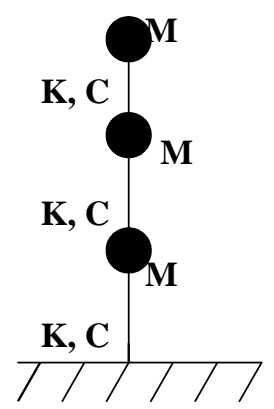

Fig. 1. Three DOF system.

$$
\begin{aligned}
& {\left[P^{(1)}\right]=} {\left[P^{(1)}\right]+\left(\left[I_{n}\right]+\left[T_{a}\right]\right)\left[P^{(1)}\right] } \\
&\left(\left[I_{n}\right]+\left[T_{a}\right]\right)^{T}+t t \cdot\left[P^{(0)}\right] \\
& {\left[P^{(0)}\right]=} {\left[P^{(0)}\right]+\left(\left[I_{n}\right]+\left[T_{a}\right]\right)\left[P^{(0)}\right] } \\
&\left(\left[I_{n}\right]+\left[T_{a}\right]\right)^{T} \\
& {\left[T_{a}\right]=\left[T_{a}\right] * 2+\left[T_{a}\right] *\left[T_{a}\right] ; t t=2 * t t ; }
\end{aligned}
$$

To avoid possible numerical ill-conditioning, it is advisable that the above equations are used only for the first $N / 2$ iterations; while for the last $N / 2$ iterations, the matrix $\left[\Phi_{n}\right]$ is used to replace $\left(\left[I_{n}\right]+\left[T_{a}\right]\right)$, and correspondingly the above equation $\left[T_{a}\right]=\left[T_{a}\right] * 2+$ $\left[T_{a}\right] *\left[T_{a}\right]$ should be replaced by $\left[\Phi_{n}\right]=\left[\Phi_{n}\right] \cdot\left[\Phi_{n}\right]$.

Thus, matrices $\left[P_{1}^{(1)}\right]\left[P_{1}^{(0)}\right]$ at time $\tau$ have been produced. Denote

$$
\begin{aligned}
& {\left[P_{1}^{(1)}\right]=\left[P^{(1)}(\tau)\right] ;} \\
& {\left[P_{1}^{(0)}\right]=\left[P^{(0)}(\tau)\right] ; \quad t t=\tau}
\end{aligned}
$$

4. Compute the response variance matrix at time $2 \tau$, $3 \tau, \ldots$, by executing the following computations for $k=1,2 \ldots$

$$
\begin{aligned}
{\left[P_{k+1}^{(1)}\right]=} & {\left[\Phi_{k}(\tau)\right]\left[P_{k}^{(1)}\right]\left[\Phi_{k}(\tau)\right]^{T} } \\
& +t t *\left[P_{0}(\tau)\right]+\left[P_{1}(\tau)\right] \\
{\left[P_{k+1}^{(0)}\right]=} & {\left[\Phi_{k}(\tau)\right]\left[P_{k}^{(0)}\right]\left[\Phi_{k}(\tau)\right]^{T} } \\
& +\left[P_{0}(\tau)\right]
\end{aligned}
$$

The above process is for zero-initial value problems. The linear superposition should be executed for the contribution of non-zero initial conditions. 


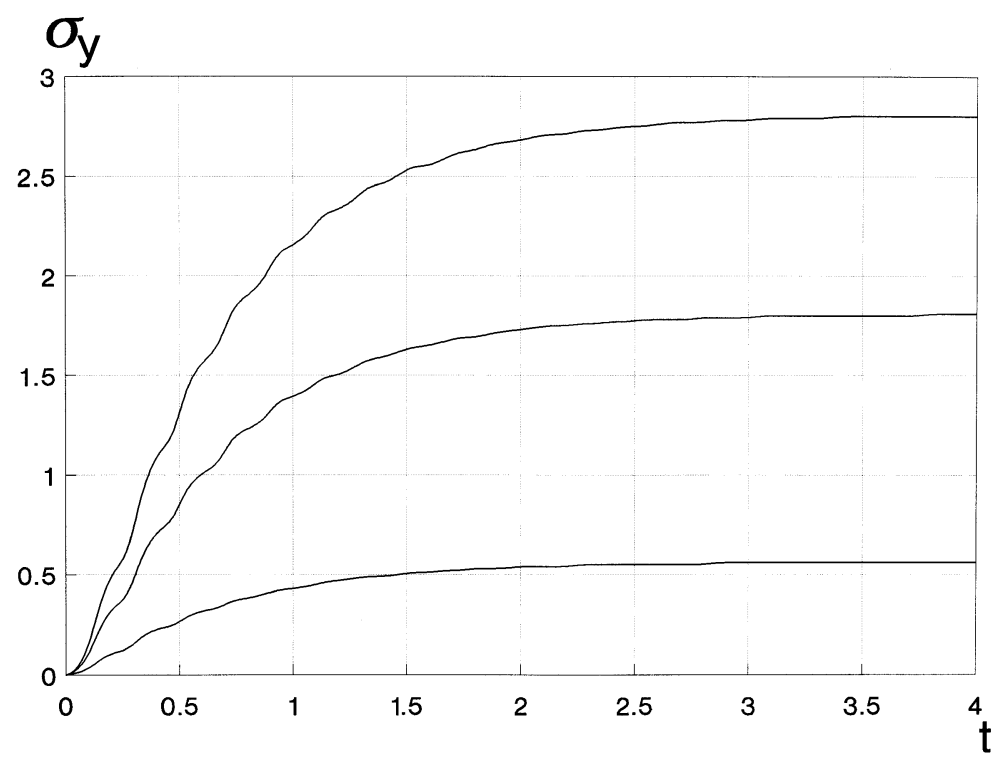

Fig. 2. Displacement variances for $g(t)=1.0$.

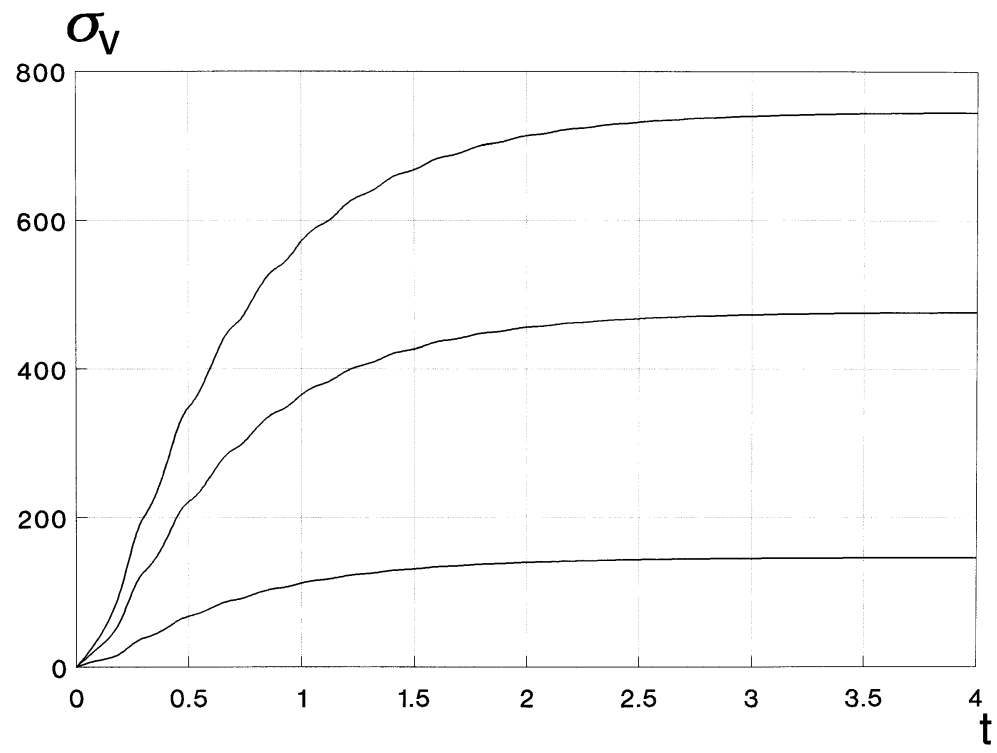

Fig. 3. Velocity variances for $g(t)=1.0$.

\section{Numerical examples}

Example 1. The equation of motion of a single degree of freedom system is

$$
\ddot{y}+2 \zeta \omega_{0} \dot{y}+\omega_{0}^{2} y=g(t) x(t)
$$

Its RHS represents a suddenly applied filtered white noise excitation, i.e. $g(t)=1.0$ (when $t \geqslant 0$ ) and the PSD of the stationary random process $x(t)$ is

$$
S_{x x}(\omega)=\frac{1+4\left(\zeta_{g} \omega / \omega_{g}\right)^{2}}{\left(1-\omega^{2} / \omega_{g}^{2}\right)^{2}+4\left(\zeta_{g} \omega / \omega_{g}\right)^{2}} S_{0}
$$

For simplicity, dimensionless parameters are used here. And it is assumed that $\omega_{=} \omega_{g}=1.0, \zeta=\zeta_{g}=0.5$ and $S_{0}=1.0$. For this simple example, analytical solutions are available [9], i.e. the variances of $y$ and $\dot{y}$ are $P_{11}=1.5 \pi=4.71238898 \ldots$ and $P_{22}=\pi=$ $3.14159265 \ldots$ 


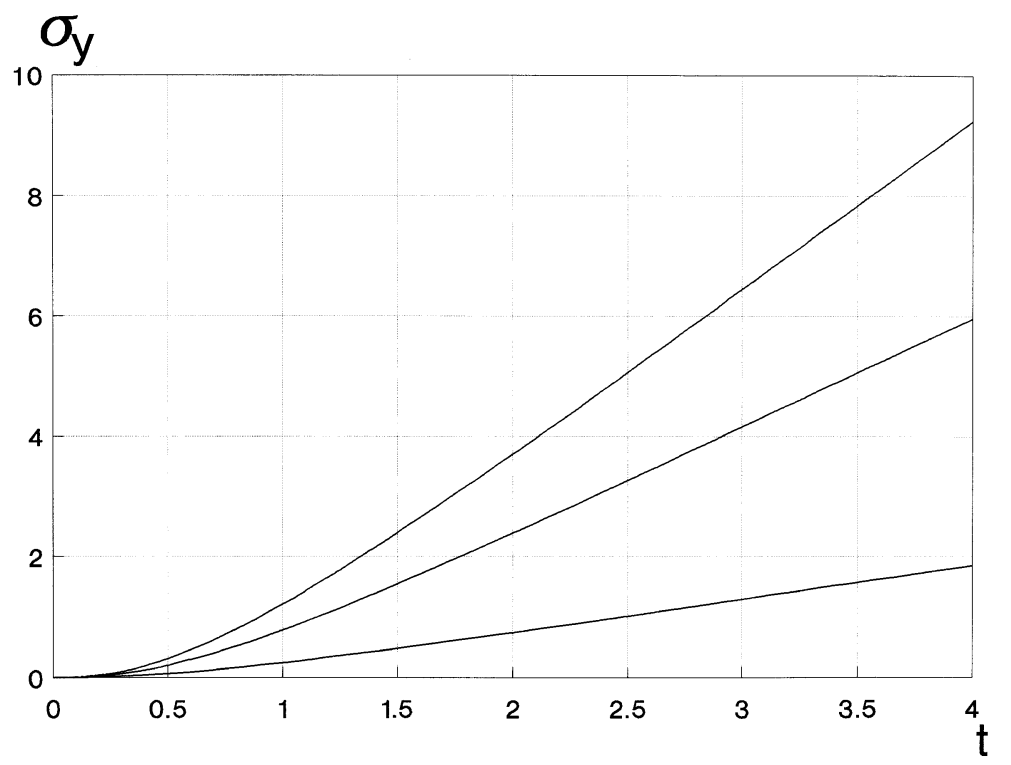

Fig. 4. Displacement variances for $g(t)=\sqrt{t}$.

Table 1

Comparison of computational precision and time

\begin{tabular}{lcccc}
\hline Method used & Time step & $\sigma_{y_{3}}^{2}$ & Error & CPU(s) \\
\hline Present paper & 0.02 & 2.803 & $0.0 \%$ & 16 \\
HPD-S & 0.1 & 2.803 & $0.0 \%$ & 226 \\
HPD-L & 0.02 & 2.778 & $0.9 \%$ & 434 \\
Newmark & 0.01 & 2.786 & $1.0 \%$ & 657 \\
Duhamel & 0.05 & 2.887 & $4.5 \%$ & 647 \\
\hline
\end{tabular}

This example has also been computed in terms of the proposed scheme. Note that when the response variances at time $\tau$ have been obtained (in the computation, $\tau$ was arbitrarily taken as 0.0015707963$)$; the doublestep formula, Eq. (24), was repeatedly used to compute the response variances at times $2 \tau, 4 \tau, 8 \tau, \ldots$ until convergence is achieved. Through only 14 such passes, i.e. at $t=16384 \tau$, the responses reach the stable values $P_{11}=4.71238898 \ldots$ and $P_{22}=3.14159265 \ldots$. Clearly, their first 9 effective digits are both identical to those of the analytical solutions.

Example 2. Consider the 3 DOF system of Fig. 1 in which $M=10.2, C=85.0$ and $K=14000.0$. Its mass, damping and stiffness matrices are, respectively

$$
\begin{aligned}
& {[M]=\left[\begin{array}{ccc}
10.2 & 0 & 0 \\
0 & 10.2 & 0 \\
0 & 0 & 10.2
\end{array}\right],} \\
& {[C]=\left[\begin{array}{ccc}
170 & -85 & 0 \\
-85 & 170 & -85 \\
0 & -85 & 85
\end{array}\right],}
\end{aligned}
$$

$$
[K]=\left[\begin{array}{ccc}
28000 & -14000 & 0 \\
-14000 & 28000 & -14000 \\
0 & -14000 & 14000
\end{array}\right]
$$

Assume that the system is subjected to a suddenly applied filtered white noise excitation, i.e. when $t \geqslant 0$,

$$
\{f(t)\}=10.2 g(t)\left\{\begin{array}{l}
1 \\
1 \\
1
\end{array}\right\} x(t)
$$

where the PSD of $x(t)$ has the form of Eq. (37) with $S_{0}=142.75, \omega_{g}=19.07$ and $\zeta_{g}=0.544$.

The integration step size was $\tau=0.02$ and 200 steps were computed. The displacement and velocity variance curves of the three masses are shown in Figs 2 and 3 . Computation shows that when $t=4.0$, the variances of such displacements are $\{0.5605,1.807,2.803\}$. This example was previously computed [7] where the pseudo excitation method was used; and when $t=4.0$, the first four digits of these variances are still $\{0.56051 .8072 .803\}$, exactly the same as those computed here. Some differences occur after their fourth digits because a limited frequency band $\omega \in[-200,200]$ was used previously [7], while the variances based on the Lyapunov equation correspond to the integration frequency band $\omega \in(-\infty, \infty)$.

Four different methods have been used [7] to compute the present example. The precision and CPU time used for all the five methods (including the present one) are listed in Table 1. It can be seen that previously the best way is the HPD-S method which used 226 


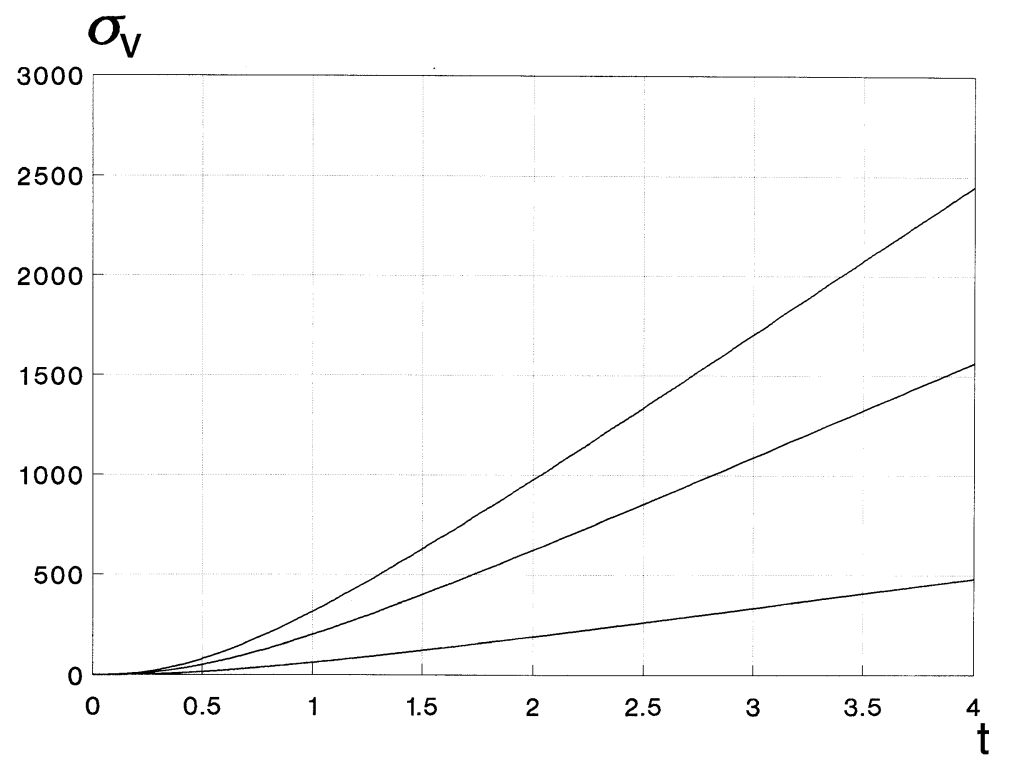

Fig. 5. Velocity variances for $g(t)=\sqrt{t}$.

$\mathrm{s}(\mathrm{CPU})$ to obtail the accurate result for $\sigma_{y_{3}}^{2}$. However, the method proposed in the present paper used only $16 \mathrm{~s}$ to produce the same result. All computations were executed on a 586 personal computer with main frequency $133 \mathrm{MHz}$.

For this example, if the modulation function is replaced by $g(t)=\sqrt{t}$ (when $t \geqslant 0)$, and repeat the above computations, the corresponding results are shown in Figs 4 and 5. Comparing Fig. 2 and Fig. 4 shows that when $t \leqslant 1$, the responses due to $g(t)=\sqrt{t}$ are obviously smaller than those due to $g(t)=1.0$. When $t \geqslant 2$, however, inverse phenomenon takes place. That is because the responses due to $g(t)=\sqrt{t}$ increase much faster after $t>1$ and so they demonstrate much stronger non-stationary property. The CPU used was $19 \mathrm{~s}$, about $20 \%$ more than that for $g(t)=1.0$.

Although only proportional damping matrix $[C]$ was used in the above, the process would be all the same if any non-proportional damping matrix is used.

\section{Conclusions}

The solution of algebraic or differential Lyapunov equations is a basic problem in the random vibration field. This problem has long been considered as very difficult to resolve. Based on the previously developed precise integration method, i.e. its HPD-L and HPD-S forms, the authors now further propose a new precise integration scheme, which can solve such Lyapunov equations accurately and very efficiently. The excitations can be stationary or non-stationary (evolutionary), white or colored random noise, therefore the proposed method applies to a wide range of problems.

\section{Acknowledgements}

The authors are grateful for the support of the NSFC funding (No. 19772009) and NKBRSF funding (No. G1999032805).

\section{References}

[1] Bendat, J.S. and Piersol, A.G., Engineering Applications of Correlation and Spectral Analysis, John Wiley, New York, 1980.

[2] Cai, S.F., Theory of Random Control, Press of Shanghai JiaoTong University, Shanghai, 1987.

[3] Lin, J.H., Zhang W.S. and Li, J.J., Structural Responses to Arbitrarily Coherent Stationary Random Vibration, Computers \& Structures 50 (1994), 629-633.

[4] Lin, J.H., Zhang, W.S. and Williams, F.W., Pseudo-excitation algorithm for nonstationary random seismic responses, Engineering Structures 16 (1994), 270-276.

[5] Lin, J.H., Li, J.J., Zhang, W.S. and Williams, F.W., Random Seismic Responses of Multi-support Structures in Evolutionary Inhomogeneous Random Fields, Earthquake Engineering and Structural Dynamics 26 (1997), 135-145.

[6] Lin, J.H., Sun, D.K., Sun, Y. and Williams, F.W., Structural Responses to Non-uniformly Modulated Evolutionary Random Seismic Excitations, Communications in Numerical Methods in Engineering 13 (1997), 605-616. 
[7] Lin, J.H., Shen, W.P. and Williams, F.W., Accurate High-speed Computation of Non-stationary Random Structural Response, Engineering Structures 19 (1997), 586-593.

[8] Lin, Y.K., Probabilistic Theory of Structural dynamics, McGraw Hill, New York, 1967.

[9] Soong, T.T. and Grigoriu, M., Random Vibration of Mechanical and Structural Systems, Prentice-Hall International, Inc., Englewoods Cliffs, New Jersey, 1993.

[10] Zhong, X.N., The precise integration method for stochastic differential equation (and closed-form solution for Lyapunov differential equation), MMM-7th Conference, Proceedings of Modern Math. And Mech., Shanghai, Nov. 26-28, 1997.
[11] Zhong, W.X., Computational Structural Mechanics and Optimal Control, Dalian University of Technology Press, Dalian, China, 1993.

[12] Zhong, W.X. and Williams, F.W., A Precise Time Step Integration Method, Proc. Inst. Mech. Engrs 208C (1995), 427430.

[13] Zhong, W.X., A New Systematic Methodology for Theory of Elasticity, Dalian University of Technology Press, Dalian, China, 1995.

[14] Zhu, W.Q., Random Vibration (in Chinese), Science Press, Beijing, 1992. 

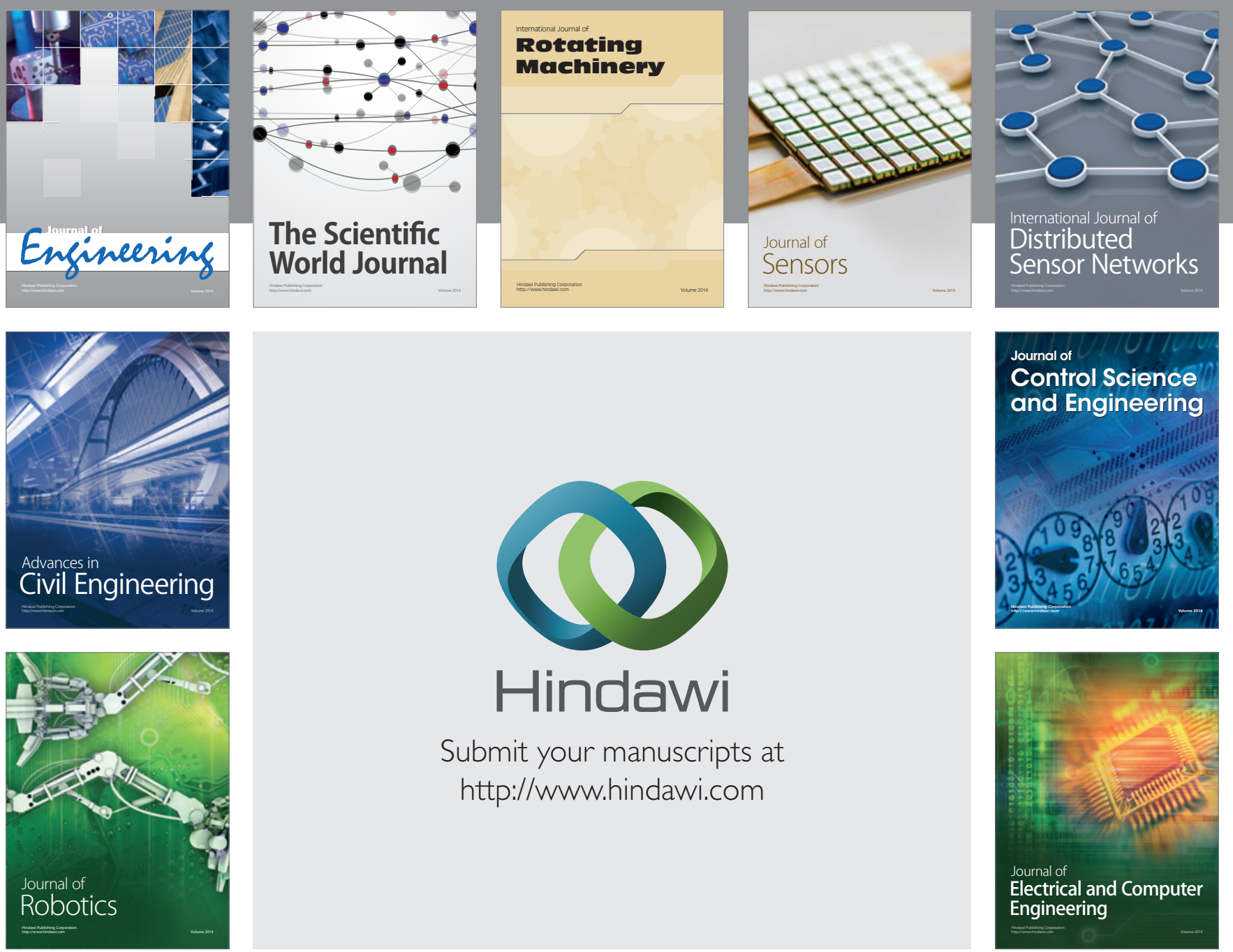

Submit your manuscripts at

http://www.hindawi.com
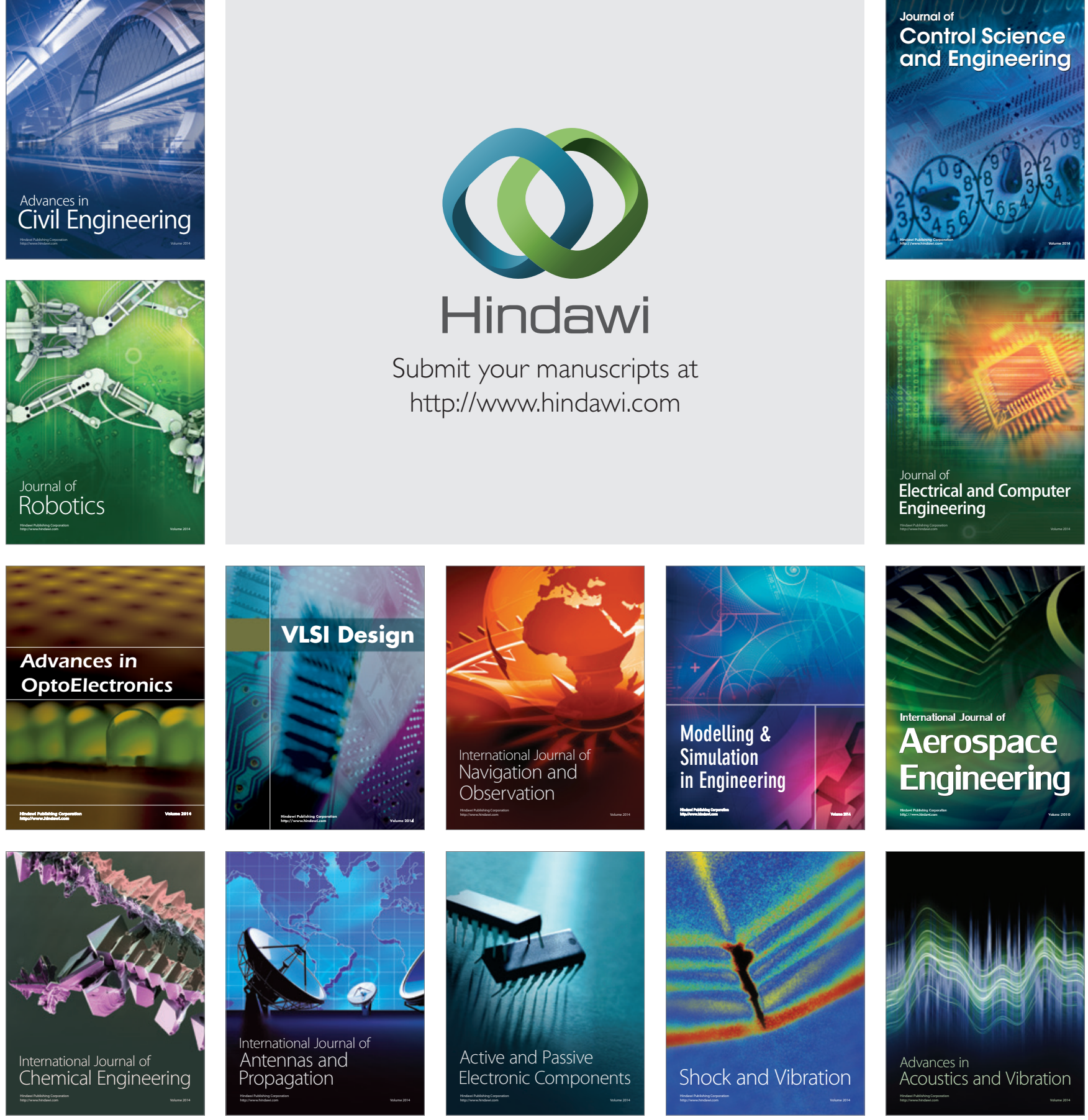